\title{
Perioperative anesthetic management for cesarean delivery of severe Wilson's disease with liver failure: a case report
}

\author{
Kana Saito ${ }^{1,2}$, Eiko Onishi ${ }^{*}$, Jun Itagaki ${ }^{1}$, Noriko Toda ${ }^{1}$, Azusa Haitani ${ }^{1}$ and Masanori Yamauchi ${ }^{1}$
}

\begin{abstract}
Background: Wilson's disease is a rare autosomal recessive disorder affecting copper metabolism, which presents liver and brain dysfunction caused by abnormal copper accumulation. We report a patient who showed exacerbation of liver failure during pregnancy.

Case presentation: A 24-year-old woman with Wilson's disease was scheduled for emergency cesarean delivery at 30 weeks of gestation. The patient exhibited severe coagulopathy and prominent body weight gain $(+30 \mathrm{~kg})$ caused by systemic edema and ascites. We decided to perform emergency cesarean delivery under general anesthesia. We used platelet concentrates, cryoprecipitate, and fibrinogen concentrate. Intraoperative hemorrhage was well controlled. On the 15th postpartum day, weight was reduced by $20 \mathrm{~kg}$ and liver function had improved. She and her baby were discharged without complications.

Conclusions: The appropriate continued treatment of Wilson's disease and supplementation of coagulation factors and/or platelets when indicated greatly increase the likelihood of a successful pregnancy, even in patients with liver failure exacerbation.
\end{abstract}

Keywords: Wilson's disease, Coagulopathy, Cesarean delivery, Rotational thromboelastometry

\section{Background}

Wilson's disease (WD) is a rare autosomal recessive disorder whose main symptom is abnormal copper accumulation mainly in the liver and brain [1,2]. WD shows a wide range of clinical manifestations, including hepatic disease, neurological abnormality, psychiatric disorder, and Kayser-Fleischer rings (copper deposition on the periphery of the cornea) [3]. Liver damage induces chronic hepatitis, liver cirrhosis, and fulminant hepatic failure, causing severe coagulopathy, encephalopathy, and hemolytic anemia.

Several reports on pregnant patients with WD have been reported, wherein patients with well-controlled WD have had successful pregnancy outcomes with continued disease treatment and close monitoring [1, 2, 47]. In recent retrospective study of pregnancy in WD, liver dysfunction during pregnancy has been observed in

\footnotetext{
* Correspondence: e-onishi@med.tohoku.ac.jp

${ }^{1}$ Department of Anesthesiology, Tohoku University Hospital, 1-1

Seiryo-machi, Aoba-ku, Sendai, Miyagi 980-8574, Japan

Full list of author information is available at the end of the article
}

$6 \%$ of patients, and it had normalized after delivery [7]. However, the anesthetic management of severe WD with pregnancy has not been reported.

We report a patient showing liver failure exacerbation during pregnancy. She was at high risk of peripartum hemorrhage due to thrombocytopenia, hypofibrinogenemia, and obstetric disseminated intravascular coagulation (DIC). She also showed a gain of $30 \mathrm{~kg}$ body weight and dyspnea due to severe systemic edema and ascites. Preoperative platelet transfusion and supplemental coagulation factor including cryoprecipitate and fibrinogen concentrate were effective to prevent peripartum hemorrhage and to avoid a large volume of transfusion.

\section{Case presentation}

A 24-year-old woman (height, $169.2 \mathrm{~cm}$; body weight, $79.2 \mathrm{~kg}$ ) with WD was scheduled for emergency cesarean delivery at 30 weeks of gestation.

The patient was diagnosed with WD at 3 years of age. Her liver function had deteriorated severely enough to require liver transplantation at 20 years of age. However, 
anti-copper therapy improved her liver function and was continued during pregnancy. She was hospitalized at 25 weeks of gestation for thrombocytopenia and fetal growth restriction (FGR) caused by placental insufficiency. Aspartate aminotransferase (AST) and alanine aminotransferase (ALT) serum levels were normalized during pregnancy, while antithrombin III (AT-III) levels were significantly lower (Table 1). On hematological evaluation, performing anticoagulation therapy with ATIII concentrate was suggested to prevent thrombophiliainduced placental dysfunction.

The patient's body weight had rapidly increased by 1 $\mathrm{kg} /$ day with marked ascites and severe edema. She experienced dyspnea and could not ambulate due to dramatic weight gain. She was also diagnosed with obstetric DIC presenting with oliguria, thrombocytopenia, respiratory distress, liver dysfunction, and severe coagulopathy [8]. At 30 weeks of gestation, emergency cesarean delivery was performed under general anesthesia to improve her liver function. We performed supplementation with 10 units of platelet transfusion, 3 packs of cryoprecipitate, and $3 \mathrm{~g}$ fibrinogen concentrate.

In the operating room, a $16 \mathrm{G}$ venous catheter was inserted. We used rotational thromboelastometry (ROTEM $^{\circ}$ sigma, Tem Innovations $\mathrm{GmbH}$, Munich, Germany), which showed normal coagulation function (Table 2). Under standard monitoring and bispectral index, we performed rapid sequence induction using $130 \mathrm{mg}$ propofol, $0.05 \mathrm{mg}$ remifentanil, and $70 \mathrm{mg}$ rocuronium. Because her face and tongue were edematous and the Mallampati classification was III, intubation difficulties were expected. Therefore, we used a video laryngoscope (McGRATH MAC\#3) for tracheal intubation. We maintained with oxygen in nitrous oxide (2:3) and $0.7 \%$ sevoflurane until delivery.

After delivery, the baby (1173 g) showed respiratory distress. Tracheal intubation and administration of intratracheal lung surfactant were performed. One- and 5min Apgar scores were 2 and 5, respectively. The neonatologists performed mechanical ventilation management for 2 days after delivery. The infant was discharged without further complications.

After delivery, continuous intravenous administration of propofol and remifentanil was started. Intraoperatively, $0.5 \mathrm{mg}$ intravenous fentanyl was administered, and continuous fentanyl injection was started at $0.04 \mathrm{mg} / \mathrm{h}$ for postoperative analgesia. Intraoperative hemorrhage was $1813 \mathrm{~mL}$ (including amniotic fluid), and ascites was $1000 \mathrm{~mL}$. The operation time was $61 \mathrm{~min}$, and anesthesia time was $179 \mathrm{~min}$. Urine volume was $80 \mathrm{~mL}$, and infusion volume was $1954 \mathrm{~mL}(654 \mathrm{~mL}$ of crystalloids and $1300 \mathrm{~mL}$ of colloids). The patient also received 3 units of red blood cells and 6 units of fresh frozen plasma (FFP). The operation was completed without massive bleeding. However, a postoperative chest X-ray indicated pulmonary edema. We decided to continue mechanical ventilation in the intensive care unit (ICU). Albumin and diuretic administration improved the patient's oxygenation and allowed for extubation the next day. She was transferred to the obstetrics ward 3 days postoperatively.

Table 1 Peripartum data of liver function, coagulation, copper metabolism and body weight

\begin{tabular}{|c|c|c|c|c|c|c|c|c|c|c|}
\hline \multirow{3}{*}{ Hemoglobin } & \multirow[b]{3}{*}{$\mathrm{g} / \mathrm{dL}$} & \multirow{3}{*}{$\begin{array}{l}\text { Normal } \\
\text { Ranges } \\
11.6-14.8\end{array}$} & \multicolumn{3}{|c|}{ Gestation weeks } & \multicolumn{5}{|c|}{ Before CS $\quad$ After CS } \\
\hline & & & \multirow{2}{*}{$\begin{array}{l}25 \text { weeks } \\
13.2\end{array}$} & \multirow{2}{*}{$\begin{array}{l}29 \text { weeks } \\
12.7\end{array}$} & \multirow{2}{*}{$\begin{array}{l}30 \text { weeks } \\
11.7\end{array}$} & \multicolumn{2}{|c|}{30 weeks and 3 days } & \multirow{2}{*}{$\begin{array}{l}\text { POD1 } \\
9.8\end{array}$} & \multirow{2}{*}{$\begin{array}{l}\text { POD4 } \\
10.5\end{array}$} & \multirow{2}{*}{$\frac{\text { POD 4C }}{11.3}$} \\
\hline & & & & & & 10.1 & 8.2 & & & \\
\hline Platelets & $\times 10^{3} / \mu \mathrm{L}$ & $158-348$ & 78 & 70 & 57 & 101 & 72 & 75 & 65 & 143 \\
\hline Total bilirubin & $\mathrm{mg} / \mathrm{dL}$ & $0.4-1.5$ & 0.8 & 0.7 & 1.0 & 0.8 & 0.6 & 0.8 & 1.4 & 1.2 \\
\hline Albumin & $\mathrm{g} / \mathrm{dL}$ & $4.1-5.1$ & 2.8 & 2.0 & 2.5 & 3.0 & 2.0 & 2.7 & 2.6 & 4.2 \\
\hline AST & $\mathrm{IU} / \mathrm{L}$ & $13-30$ & 37 & 31 & 25 & 21 & 16 & 25 & 26 & 40 \\
\hline ALT & $\mathrm{IU} / \mathrm{L}$ & $7-23$ & 27 & 19 & 14 & 14 & 9 & 11 & 12 & 29 \\
\hline ChE & $U / L$ & $201-421$ & 52 & 43 & 33 & & & 89 & 86 & 109 \\
\hline PT & $\%$ & $80-127$ & 79.6 & 66.8 & 52.7 & 49.6 & 43.6 & 46.5 & 46.7 & 59.9 \\
\hline PT-INR & & $<1.15$ & 1.11 & 1.2 & 1.31 & 1.38 & 1.46 & 1.42 & 1.40 & 1.26 \\
\hline APTT & $\mathrm{sec}$ & 26.9-38.1 & 37.4 & 44.8 & 50.2 & 38.4 & 52.8 & 48.3 & 56.3 & 36.2 \\
\hline Fibrinogen & $g / L$ & $2.00-4.00$ & 2.01 & 1.55 & 1.24 & 1.30 & 1.30 & 1.12 & 1.27 & 1.37 \\
\hline Antithrombin III & $\%$ & $80-130$ & 36 & 72 & 41 & 56 & 38 & 60 & 43 & 47 \\
\hline Serum copper & $\mu \mathrm{g} / \mathrm{dL}$ & $66-130$ & 34 & & & & & & 28 & 45 \\
\hline Ceruloplasmin & $\mathrm{mg} / \mathrm{dL}$ & $21-37$ & 6.2 & & & & & & 6.6 & 8.3 \\
\hline Body Weight & $\mathrm{kg}$ & $49^{\mathrm{a}}$ & 59.3 & 70 & 76.1 & 79.1 & 77.2 & 77.8 & 69.1 & 60.8 \\
\hline
\end{tabular}

CS cesarean section, $P O D$ postoperative day, AST aspartate aminotransferase, ALT alanine aminotransferas, ChE cholinesterase, $P T$ prothrombin time, INR international normalized ratio, $A P T T$ activated partial thromboplastin time abefore pregnancy 
Table 2 ROTEM results before cesarean delivery

\begin{tabular}{lllll}
\hline & FIBTEM (normal ranges) & EXTEM (normal ranges) & INTEM (normal ranges) & APTEM (normal ranges) \\
\hline CT, seconds & $59(46-84)$ & $54(50-80)$ & $142(161-204)$ & $53(41-80)$ \\
CFT, seconds & & $108(46-149)$ & $108(62-130)$ & $112(62-184)$ \\
Alpha angle, & & $72(63-83)$ & $73(66-77)$ & $75(60-80)$ \\
A5, mm & $10(5-20)$ & $36(32-52)$ & $35(33-52)$ & $35(28-50)$ \\
A10, mm & $10(6-21)$ & $46(43-63)$ & $45(43-62)$ & $45(39-61)$ \\
A20, mm & $10(6-21)$ & $53(52-70)$ & $52(50-68)$ & $51(48-68)$ \\
\hline
\end{tabular}

CT clotting time, CFT clot formation time, Alpha angle the angle of tangent between the centerline and the curve through the 20-mm amplitude point, $A 5$ amplitude at $5 \mathrm{~min}, A 10$ amplitude at $10 \mathrm{~min}, A 20$ amplitude at $20 \mathrm{~min}$, EXTEM the extrinsic coagulation pathway, INTEM the intrinsic coagulation pathway, FIBTEM the quality of the fibrin-based clot, APTEM the detection of hyperfibrinolysis

On the 15th postpartum day, her body weight showed a decrease of $20 \mathrm{~kg}$, and she was discharged from the hospital. Subsequently, coagulation status was improved to pre-pregnancy levels 40 days after delivery.

\section{Discussion}

WD shows abnormal copper accumulation in the liver and brain due to a decrease in ceruloplasmin, which is the transport protein bound to serum copper. ATP7B mutation on chromosome 13q14 gene has been identified as the causative gene [1,2]. Generally, the treatment of WD is by pharmaceutical means that reduce copper absorption or promote copper excretion. Liver transplantation may also be performed in severe liver failure that is uncontrolled by pharmaceutical therapy.

Our patient had severe WD controlled with anticopper therapy. Her liver function had rapidly deteriorated during pregnancy, and she showed severe coagulopathy, thrombocytopenia, and obstetric DIC. Intensive coagulation therapy could prevent massive peripartum hemorrhage.

Fibrinogen was reported to be the most important factor in obstetric hemorrhage [9]. It has been reported that a fibrinogen concentration $\leq 2 \mathrm{~g} / \mathrm{L}$ has a positive predictive value of $100 \%$ for progression to severe postpartum hemorrhage [10]. Fibrinogen supplementation for postpartum hemorrhage has been reported [9-12]; however, prophylactic administration of fibrinogen in severe coagulopathy has not been well investigated. Our patient experienced both hypofibrinogenemia and thrombocytopenia, and was diagnosed with obstetric DIC. Her preoperative serum fibrinogen had decreased to $<1.5 \mathrm{~g} / \mathrm{L}$. Therefore, we considered the risk of massive perioperative hemorrhage to be high and that a massive blood transfusion may be required. The patient's dramatic body weight gain had caused dyspnea and severe systemic edema. We were thus concerned about the cardiopulmonary dysfunction due to volume load caused by massive blood transfusion and fluid shift from prominent systemic edema. A large FFP transfusion can induce pulmonary edema due to volume overload [13]. Therefore, we decided to administer cryoprecipitate and fibrinogen concentrate. Additionally, we confirmed her coagulation function with ROTEM to determine the appropriate dose of fibrinogen concentrate. Although there have been reports about baseline ROTEM parameters of healthy pregnant women [14], ROTEM analysis in obstetrics has not been established. The efficacy of ROTEMguided fibrinogen therapy for postpartum hemorrhage has been reported $[15,16]$. In our case, her blood examination on the day of surgery showed thrombocytopenia, hypofibrinogenemia, and coagulation disorder. Preoperative platelet transfusion, cryoprecipitate, and fibrinogen concentrate administration improved her coagulation function and helped to avoid massive hemorrhage intraoperatively. Coagulation monitoring by ROTEM allowed us to evaluate her coagulation function just before surgery and determine the dose of supplemental fibrinogen administration.

WD exacerbation, as seen in our patient, is rare; therefore, we investigated other factors that can cause acute liver dysfunction associated with pregnancy, including the HELLP syndrome, acute fatty liver of pregnancy, and exacerbation of underlying chronic liver disease [17]. There are case reports of pregnant women with WD who had developed placenta abruption [18], preeclampsia [6], or HELLP syndrome [19, 20]. In our case, levels of total bilirubin, AST, and ALT were within normal limits. Therefore, liver function deterioration was considered to be due to WD exacerbation. However, the prominent systemic edema and significant weight gain might have also been attributed to a preeclampsiarelated condition. Additionally, thrombocytopenia, decrease in urine output, and severe coagulopathy in the third trimester indicated obstetric DIC [12], which made her pregnancy difficult to continue.

Our patient had an episode of serious hepatic insufficiency at 20 years of age due to discontinuation of D-penicillamine. Discontinuation of medication in WD has been reported to exacerbate liver function and liver failure [21]. Liver biopsy revealed hepatic fibrosis, and liver function had already deteriorated severely enough to necessitate liver transplantation. Poor hepatic reserve may have been irreversible, despite copper metabolism and liver function 
showing improvement by treatment. She showed a remarkable decrease in liver function including albumin, AT-III, and coagulation factor levels, while AST and ALT levels remained normal. No reports show that only protein synthesis function markedly decreases in WD. Pregnancy might induce unexpected liver dysfunction in patients with WD, even when WD treatment is ongoing.

\section{Conclusion}

We showed the successful management of pregnancy by cesarean delivery in a patient with WD. The exacerbation of WD during pregnancy caused severe coagulopathy, prominent body weight gain, respiratory distress, and placental insufficiency. Supplementation of coagulation factor before surgery allowed us to manage general anesthesia without massive bleeding. Both the mother and her baby were stable postoperatively, and the mother's liver function recovered after delivery. Multidisciplinary therapy during pregnancy is critical for WD patients with liver failure. The appropriate continued treatment of WD, including pharmaceutical treatment and coagulation therapy, greatly increases the likelihood of a successful pregnancy, even in patients with liver failure exacerbation.

\section{Abbreviations}

ALT: Alanine aminotransferase; APTT: Activated partial thromboplastin time; AST: Aspartate aminotransferase; AT-III: Antithrombin III; CFT: Clot formation time; ChE: Cholinesterase; CS: Cesarean delivery; CT: Clotting time; DIC: Disseminated intravascular coagulation; FFP: Fresh frozen plasma; FGR: Fetal growth restriction; ICU: Intensive care unit; INR: International normalized ratio; POD: Postoperative day; PT: Prothrombin time; ROTEM: Rotational thromboelastometry; WD: Wilson's disease

\section{Acknowledgements}

We would like to thank Editage (www.editage.com) for English language editing.

\section{Authors' contributions}

$\mathrm{KS}, \mathrm{EO}$, and $\mathrm{AH}$ performed the anesthetic management. NT supervised the anesthetic management. Jl researched about the peripartum management of Wilson's disease. KS wrote the manuscript. MY reviewed the manuscript. The final version of the manuscript was approved by all authors.

\section{Funding}

This research did not receive any specific grant from funding agencies in the public, commercial, or not-for-profit sectors.

\section{Availability of data and materials \\ Not applicable}

Ethics approval and consent to participate

Not applicable

\section{Consent for publication}

We had obtained the consent to publish from the patient.

\section{Competing interests}

The authors declare that they have no competing interests.

\section{Author details}

'Department of Anesthesiology, Tohoku University Hospital, 1-1 Seiryo-machi, Aoba-ku, Sendai, Miyagi 980-8574, Japan. ${ }^{2}$ Department of Anesthesiology, Tohoku Rosai Hospital, 4-3-21 Dainohara, Aoba-ku, Sendai, Miyagi 981-8563, Japan.
Received: 31 August 2019 Accepted: 15 October 2019

Published online: 13 November 2019

\section{References}

1. Mustafa MS, Shamina AH. Five successful deliveries following 9 consecutive spontaneous abortions in a patient with Wilson disease. Aust N Z J Obstet Gynaecol. 1998;38:312-4

2. Wan $Y$, Jiang $X$, Lin $X$. Anesthetic management of cesarean delivery for a parturient with Wilson's disease: a case report. Medicine (Baltimore). 2018; 97:e10454.

3. Ala A, Walker AP, Ashkan K, Dooley JS, Schilsky ML. Wilson's disease. Lancet 2007:369:397-408

4. Furman B, Bashiri A, Wiznitzer A, Erez O, Holcberg G, Mazor M. Wilson's disease in pregnancy: five successful consecutive pregnancies of the same woman. Eur J Obstet Gynecol Reprod Biol. 2001:96:232-4.

5. Lee HJ, Seong WJ, Hong SY, Bae JY. Successful pregnancy outcome in a Korean patient with symptomatic Wilson's disease. Obstet Gynecol Sci. 2015:58:409-13.

6. Malik A, Khawaja A, Sheikh L. Wilson's disease in pregnancy: case series and review of literature. BMC Res Notes. 2013;6:421.

7. Pfeiffenberger J, Beinhardt S, Gotthardt DN, et al. Pregnancy in Wilson's disease: management and outcome. Hepatology. 2018:67:1261-9.

8. Kobayashi T. Obstetrical disseminated intravascular coagulation score. J Obstet Gynaecol Res. 2014:40:1500-6.

9. Seto S, Itakura A, Okagaki R, Suzuki M, Ishihara O. An algorithm for the management of coagulopathy from postpartum hemorrhage, using fibrinogen concentrate as first-line therapy. Int J Obstet Anesth. 2017;32:116.

10. McDonnell NJ, Browning R. How to replace fibrinogen in postpartum haemorrhage situations? (Hint: Don't use FFP!). Int J Obstet Anesth. 2018;33: 4-7.

11. McQuilten ZK, Bailey M, Cameron PA, et al. Fibrinogen concentration and use of fibrinogen supplementation with cryoprecipitate in patients with critical bleeding receiving massive transfusion: a bi-national cohort study. $\mathrm{Br}$ Haematol. 2017:179:131-41.

12. Nascimento B, Goodnough LT, Levy JH. Cryoprecipitate therapy. Br J Anaesth. 2014:113:922-34.

13. Era $S$, Matsunaga $S$, Matsumura $H$, Murayama $Y$, Takai $Y$, Seki $H$. Usefulness of shock indicators for determining the need for blood transfusion after massive obstetric hemorrhage. J Obstet Gynaecol Res. 2015;41:39-43.

14. Lee J, Eley VA, Wyssusek KH, et al. Baseline parameters for rotational thromboelastometry $(\mathrm{ROTEM}(\mathrm{R}))$ in healthy women undergoing elective caesarean delivery: a prospective observational study in Australia. Int J Obstet Anesth. 2019:38:10-8.

15. McNamara H, Mallaiah S, Barclay P, Chevannes C, Bhalla A. Coagulopathy and placental abruption: changing management with ROTEM-guided fibrinogen concentrate therapy. Int J Obstet Anesth. 2015;24:174-9.

16. Snegovskikh D, Souza D, Walton Z, et al. Point-of-care viscoelastic testing improves the outcome of pregnancies complicated by severe postpartum hemorrhage. J Clin Anesth. 2018:44:50-6.

17. Bacak SJ, Thornburg LL. Liver failure in pregnancy. Crit Care Clin. 2016;32: $61-72$

18. Theodoridis TD, Zepiridis L, Athanatos D, Dinas K, Tzevelekis F, Bontis JN Placenta abruption in a woman with Wilson's disease: a case report. Cases J. 2009:2:8699

19. Avcioglu SN, Altinkaya SO, Kucuk M, Zafer E, Demircan Sezer S, Odabasi AR. Wilson's disease presenting with HELLP syndrome; a case report. Turk J Obstet Gynecol. 2015;12:56-9.

20. Czlonkowska A, Gromadzka G, Buttner J, Chabik G. Clinical features of hemolysis, elevated liver enzymes, and low platelet count syndrome in undiagnosed Wilson disease: report of two cases. Arch Gynecol Obstet. 2010;281:129-34.

21. Roberts EA, Schilsky ML, American Association for Study of Liver Diseases (AASLD). Diagnosis and treatment of Wilson disease: an update. Hepatology. 2008:47:2089-111.

\section{Publisher's Note}

Springer Nature remains neutral with regard to jurisdictional claims in published maps and institutional affiliations. 